\title{
Extending the Real Remoteness of Long-Range Brillouin Optical Time-Domain Fiber Analyzers
}

\author{
Marcelo A. Soto, Xabier Angulo-Vinuesa, Sonia Martin-Lopez, Sang-Hoon Chin, Juan Diego Ania-Castañon, \\ Pedro Corredera, Etienne Rochat, Member, IEEE, Miguel Gonzalez-Herraez, \\ and Luc Thévenaz, Senior Member, IEEE
}

\begin{abstract}
The real remoteness of a distributed optical fiber sensor based on Brillouin optical time-domain analysis is considerably extended in this paper using seeded second-order Raman amplification and optical pulse coding. The presented analysis and the experimental results demonstrate that a proper optimization of both methods combined with a well-equalized two-sideband probe wave provide a suitable solution to enhance the signal-to-noise ratio of the measurements when an ultra-long sensing fiber is used. In particular, the implemented system is based on an extended optical fiber length, in which half of the fiber is used for sensing purposes, and the other half is used to carry the optical signals to the most distant sensing point, providing also a long fiber for distributed Raman amplification. Power levels of all signals launched into the fiber are properly optimized in order to avoid nonlinear effects, pump depletion, and especially any power imbalance between the two sidebands of the probe wave. This last issue turns out to be extremely important in ultra-long Brillouin sensing to provide strong robustness of the system against pump depletion. This way, by employing a $240 \mathrm{~km}$-long optical fiber-loop, sensing from the interrogation unit up to a $120 \mathrm{~km}$ remote position (i.e., corresponding to the real sensing distance away from the sensor unit) is experimentally demonstrated with a spatial resolution of 5 m. Furthermore, this implementation requires no powered element in the whole $240 \mathrm{~km}$ fiber loop, providing considerable advantages in situations where the sensing cable crosses large unmanned areas.
\end{abstract}

Index Terms-Brillouin scattering, distributed optic fiber sensor, distributed Raman amplification, optical pulse coding, strain and temperature measurements.

Manuscript received April 29, 2013; revised October 14, 2013 and November 8, 2013; accepted November 18, 2013. Date of current version December 6, 2013. This work was supported in part by the Spanish Ministry of Science and Innovation through projects TEC2009-14423-C02-01 and TEC200914423-C02-02 and the Comunidad de Madrid through Project FACTOTEM-2. The work of S. Martin-Lopez was supported by the Spanish Ministry of Science and Innovation through a "Juan de la Cierva" Contract. The work of M. A. Soto and L. Thévenaz was supported by the Swiss Commission for Technology and Innovation Project 13122.1. The work of M. Gonzalez-Herraez was supported by the European Research Council through Starting Grant U-FINE Grant 307441

M. A. Soto and L. Thévenaz are with the Swiss Federal Institute of Technology of Lausanne, Institute of Electrical Engineering, 1015 Lausanne, Switzerland (e-mail: marcelo.soto@epfl.ch; luc.thevenaz@epfl.ch).

X. Angulo-Vinuesa, J. D. Ania-Castañon, and P. Corredera are with the Instituto de Optica, Consejo Superior de Investigaciones Científicas, Madrid 28006 , Spain (e-mail: xabier.angulo@focustech.eu; juan.diego@io.cfmac.csic.es; pcorredera@io.cfmac.csic.es).

S. Martin-Lopez and M. Gonzalez-Herraez are with the Departamento de Electrónica, Universidad de Alcalá, Edificio Politécnico, Madrid 28871, Spain (e-mail: sonia.martinlo@uah.es; miguelg@depeca.uah.es).

S.-H. Chin and E. Rochat are with Omnisens SA, 1110 Morges, Switzerland (e-mail: sanghoon.chin@omnisens.com; etienne.rochat@omnisens.com).

Color versions of one or more of the figures in this paper are available online at http://ieeexplore.iee.org.

Digital Object Identifier 10.1109/JLT.2013.2292329

\section{INTRODUCTION}

$\mathbf{T}$ HE demand on distributed fiber optic sensors based on Brillouin optical time-domain analysis (BOTDA) [1]-[3] has been increasing over the past years in many application fields due to their unique capability to monitor distributed strain [1] and temperature [2] changes over many tens of $\mathrm{km}$ of optical fiber with metric spatial resolution. BOTDA sensors make use of two counter-propagating optical waves, a pulsed pump wave and a continuous-wave $(\mathrm{CW})$ probe signal, which interact with an acoustic wave generated within the fiber through stimulated Brillouin scattering (SBS) [3]. Since pump and probe signals must propagate in counter-propagating directions along the optical fiber, the BOTDA interrogation unit requires access to both fiber ends. Therefore, BOTDA sensors can only exploit the entire sensing fiber whenever the fiber ends are at close distance, typically in bi-dimensional or three-dimensional configurations, e.g., for monitoring civil structures; however, for applications in which a linear fiber configuration is required, e.g., for long pipelines or offshore monitoring, the real remoteness of the sensor, and hence, the useful sensing distance, is restricted to half the fiber length.

A potential scheme to increase the remoteness of the sensor is the use of a single-end-access BOTDA [4]-[6]; however, such schemes are highly subject to optical noise and provide limited sensing performance. A more suitable alternative to monitor linear structures along ultra-long distances is to double the length of the optical fiber connected to a standard two-end-access BOTDA sensing unit, while only half of the fiber is used for sensing purposes [7]. This way, the first half of the optical fiber can be used as a distributed sensing element, while the second half is only dedicated to transfer the probe signal up to the most distant point in the sensing fiber. In this paper this fiber arrangement will be hereafter called "linear sensing fiber configuration." Although the sensing length is not increased in this fiber configuration, the real remoteness of the sensor can be doubled [7], allowing the BOTDA system to sense critical points located at much longer distances. Unfortunately, with such a scheme the probe power reaching the receiver is highly attenuated, and therefore, no sensing capabilities or eventually an extremely low sensing performance should be expected at long distances.

Some techniques have been recently proposed in the literature to extend the sensing length of BOTDA sensors operating with the standard fiber configuration, challenging the wellknown trade-off between spatial resolution and sensing range. In particular, optical pulse coding [8] and distributed firstand second-order Raman amplification [9]-[11] have made it 
possible to sense ranges beyond $100 \mathrm{~km}$ with spatial resolutions of a few meters. Although the use of those methods seems to be feasible and straightforwardly applicable for a very long linear fiber configuration, the high probe attenuation and the limited sensitivity of the optical detection stage make the design and power optimization much more challenging when compared to the use of the typical fiber-loop scheme [11]. In particular, the onset of intense amplified spontaneous Brillouin scattering imposes special constraints to the maximum probe power that is allowed in the fiber since this amplified Brillouin wave induces additional loss on the probe. It is interesting to notice that, although perfect power symmetry is required between the two probe sidebands to provide strong robustness against pump depletion in a long sensing fiber [12], the impact of any potential probe power imbalance in long-range sensing has been poorly addressed in the literature so far. One possibility to increase the optical power reaching the detector is to use bi-directional in-line Erbium-doped fiber amplifiers (EDFAs) along the whole optical fiber [7], [13], partially compensating the fiber loss on both probe waves and Brillouin pump. However, in several applications for ultra-long remote sensing, such as long terrestrial pipelines or subsea flowlines, no electrical power is available for optical amplifiers. Actually, carrying the electrical power from one of the fiber-ends would require several tens or even hundreds kilometer of electrical cables, resulting in an inefficient, costly, unreliable and unsafe alternative for longrange fiber sensing. Thus, it can be considered that methods based on powered optical repeaters at the middle of the fiber length do not provide a suitable and practical solution to extend the sensing range of BOTDA systems for many ultra-long applications.

In this paper an ultra-long BOTDA sensor in a linear configuration and without any powered elements in the sensing loop is implemented based on the optimized design of a seeded second-order distributed Raman amplifier [14], [15] in combination with optical pulse coding techniques [16], [17]. While Raman amplification is used to increase the probe power beyond the minimum detectable level, pulse coding enhances the probe power contrast resulting from the Brillouin gain, thus increasing the signal-to-noise ratio (SNR) of the measurements [17]. The optimization procedure required for the system to operate along an ultra-long linear fiber configuration is fully described through this paper, paying special attention to the impact of the amplified spontaneous Brillouin scattering generated by the $\mathrm{CW}$ two-sideband probe signal. This way, the differences in the parameter optimization procedure with respect to previously published techniques [18], [19], operating with the standard fiber configuration, are clarified. The power levels of the Brillouin pump, probe signal and Raman pumps are adjusted to optimize the Brillouin interaction within the sensing fiber and to simultaneously avoid probe power imbalance, pump depletion [12] and nonlinear effects [20]. In this way, to the best of our knowledge, the first distributed measurement with a real remoteness of $120 \mathrm{~km}$, using a $240 \mathrm{~km}$-long standard singlemode fiber, is experimentally demonstrated using a BOTDA sensor.

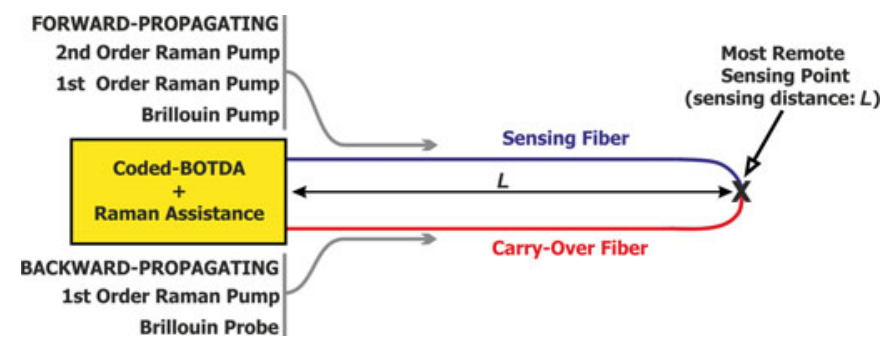

Fig. 1. Schematic diagram showing the principle of a long-range BOTDA sensor employing a linear sensing fiber configuration.

\section{EXTENDING THE REMOTENESS OF UlTRA-LONG RANGE BOTDA SENSORS}

\section{A. BOTDA Employing a Linear Sensing Fiber Configuration}

In order to increase the real physical distance that a BOTDA sensor can reach, a linear sensing fiber configuration, as the one shown in Fig. 1, can be used. In comparison with the standard reported fiber configuration, where the whole optical fiber length is used for sensing [8]-[11], in this case only half of the optical fiber length is used for sensing purpose (this is the actual sensing fiber), while the other half (denominated as carry-over fiber) is only used to transfer the probe signal up to the most remote sensing location, corresponding to the farthest end of the sensing fiber. This feature actually makes a big difference when compared to the basic fiber configuration, since in this case the probe signal cumulates twice the effect of fiber attenuation; and therefore, it is more likely that the probe power does not reach the minimum detectable level at the optical receiver stage.

Using techniques such as distributed Raman amplification and/or optical pulse coding, sensing lengths up to $120 \mathrm{~km}$ have been reported using the standard fiber-loop configuration [8]-[11]. In all those cases the full sensing range has been determined by the fiber length, and hence only half of this length constitutes the real remoteness from the interrogation unit. Consequently, the longest sensing range reported with those techniques, which is $120 \mathrm{~km}$ [8], [19], can only secure a remote sensing up to a distance of $60 \mathrm{~km}$ away from the interrogation unit. Extending the remoteness of the sensor up to $120 \mathrm{~km}$ requires a $240 \mathrm{~km}$ fiber-loop; however, under such a condition the probe signal is attenuated by about $48 \mathrm{~dB}$ while propagating along the entire optical fiber before reaching the receiver. As a consequence, the $\mathrm{CW}$ probe power at the receiver is expected to be reduced by $24 \mathrm{~dB}$ when compared to the power measured in the basic configuration (as in [8], [19]), in which only a $120 \mathrm{~km}$-long fiber-loop is used. This situation actually imposes a significant technical challenge to the design and implementation of the proposed ultra-long range BOTDA system.

It is worth mentioning that the maximum probe power inside the fiber is limited by the onset of intense amplified spontaneous Brillouin scattering, which is about $7 \mathrm{dBm}$ in standard optical fibers [12]. Therefore, in order to reach a remoteness of $120 \mathrm{~km}$, using a $240 \mathrm{~km}$-long fiber loop with no Raman amplification, the maximum probe power that can be launched into the sensing fiber is about $-17 \mathrm{dBm}$ (after $24 \mathrm{~dB}$ of attenuation within 
the carry-over fiber). This low probe power level is expected to be further reduced down to $-41 \mathrm{dBm}$ at the input of the receiver (neglecting the insertion loss of components), resulting in a power level that is difficult to detect even if a low-power preamplifier is used. In order to compensate for this additional attenuation of the probe signal, distributed Raman amplification provides a suitable solution to push the maximum power level of the pump and probe inside the fiber, allowing the system to recover about $50 \mathrm{~km}$ of sensing distance using the proposed seeded second-order Raman amplification scheme. This would allow the probe signal to reach the minimum power levels required by the pre-amplifier at the receiver (typically used for long-range sensing [8]) to produce an acceptable optical SNR (OSNR) at the photo-detector. However, the power levels involved in a long sensing fiber configuration, such as the one shown in Fig. 1, are very different to the basic scheme [19], and therefore, a dedicated optimization of the Raman amplification is required. Actually, the optimization obtained from a system operating with the standard fiber-loop configuration cannot be straightforwardly applied (i.e., linearly extrapolated) to the case in which long sensing/carry-over fibers are used. This is because nonlinear effects occurring in the carry-over fiber have a significant impact on the power distribution of the signals propagating along the useful sensing fiber. Therefore, special design and optimization are required in this long linear fiber case.

\section{B. Use of Seeded Second-Order Raman Amplification Combined With Optical Pulse Coding}

To overcome the problems associated to the sensitivity at the photo-detection stage when using very long sensing/carryover fibers, in this paper optical pulse coding and seeded second-order Raman amplification are combined together in the BOTDA sensor.

The use of first-order distributed Raman amplification combined with optical pulse coding in BOTDA sensing has been proposed by X.-H. Jia et al. in [18]; however, the use of nonoptimized Raman pump powers and the high-RIN of the Raman pumping scheme led in that case to a noisy system with a reduced sensing performance. Recently, much longer sensing distances have been achieved combining the above mentioned methods and optimizing the power levels launched into the usual standard fiber-loop configuration [19].

In the proposed scheme, the Brillouin pump is generated by intensity modulation of the laser light mapping the pulse sequence patterns defined by Simplex codes [16]. The pump power can be this way spread in the time domain, so that the intensity contrast of the Brillouin gain measurements is increased while the maximum peak pump power inside the fiber is kept below the onset of nonlinear effects, primarily modulation instability [17].

In order to improve the OSNR of the probe signal reaching the photo-detector, both the OSNR and gain resulting from the distributed Raman amplifier have to be optimized. On the one hand, in order to increase the maximum distance reached by the coded Brillouin pump, a "seeded" second-order Raman amplification scheme [14], [15] is implemented to amplify the pulse sequences. In this scheme a low-power first-order Raman pump at $1455 \mathrm{~nm}$ acts as a seed that is amplified by a high-power second-order Raman pump at $1365 \mathrm{~nm}$. Although this amplification scheme has been widely used in telecommunication systems [14], [15], to the best of our knowledge, it has never been implemented for BOTDA sensing. This way the maximum Brillouin pump power is shifted to a more distant location along the sensing fiber (up to about $30 \mathrm{~km}$ ). The sensing range extension is reasonably estimated to be about $15 \mathrm{~km}$ with respect to direct first-order Raman amplification. Both first- and secondorder Raman pump power levels have to be carefully adjusted in order to increase the power of the Brillouin pump at the end of the sensing fiber (or equivalently, to extend the distance reached by the coded pump), but at the same time, to avoid that the maximum Brillouin pump power in the fiber exceeds the threshold of nonlinear effects [20].

On the other hand, first-order low-RIN Raman amplification is also necessary on the probe side to secure a good performance of Raman-assisted BOTDA sensors [18]. Actually, although in counter-propagating Raman amplification the Raman pump RIN is averaged over the whole amplifier transit time, resulting in a negligible impact of the RIN on the sensor performance, a highly efficient pump-to-signal RIN transfer occurs in co-propagating Raman amplification [11], [21]. Therefore, the co-propagating Raman assistance in the probe side has to be performed using low-RIN semiconductor laser diodes (LDs) to minimize the pump RIN transfer to the probe signal. Second-order Raman assistance co-propagating with the probe would result in a high and efficient pump RIN transfer, degrading significantly the SNR of the measurements. For this reason, as described in Fig. 1, only first-order Raman LD pumping is here proposed to copropagate with the probe. As stated, the RIN characteristics of the Raman pumps used in the proposed second-order seeded Raman amplification scheme are not relevant. This can be easily justified by the fact that even though a high RIN transfer to the Brillouin pump is expected in this case (due to the copropagating pumping scheme [21]), no penalties are expected in the SNR of the measurements due to the low cut-off frequency of the counter-propagating pump-to-signal RIN transfer function of the Brillouin scattering process [11].

Another important aspect of the proposed scheme is the use of pump pulses with return-to-zero (RZ) modulation format. It is well-known that RZ pulses are required to apply optical pulse coding in BOTDA sensors, suppressing bit patterning effects resulting from pre-excited acoustic waves [22]. It turns out that, in the case of Raman assistance, RZ pulses also have the advantage to minimize the Raman cross-gain modulation occurring when the forward-propagating Raman pump is depleted. Actually, a pulse separation of at least $50 \mathrm{~ns}$ is normally required to secure a complete decay of the acoustic wave amplitude between pulses of the code sequence [22]. Such a time interval is much longer than the maximum walk-off between the forward Raman pump at $1450 \mathrm{~nm}$ and the Brillouin pump-probe signals at $1550 \mathrm{~nm}$ [21], which is about $16 \mathrm{~ns}$ in ultra-long standard single-mode fibers [19]. Therefore, if the co-propagating Raman pump at $1450 \mathrm{~nm}$ is depleted by the amplification of a single Brillouin pump pulse, no impact on the amplification of 


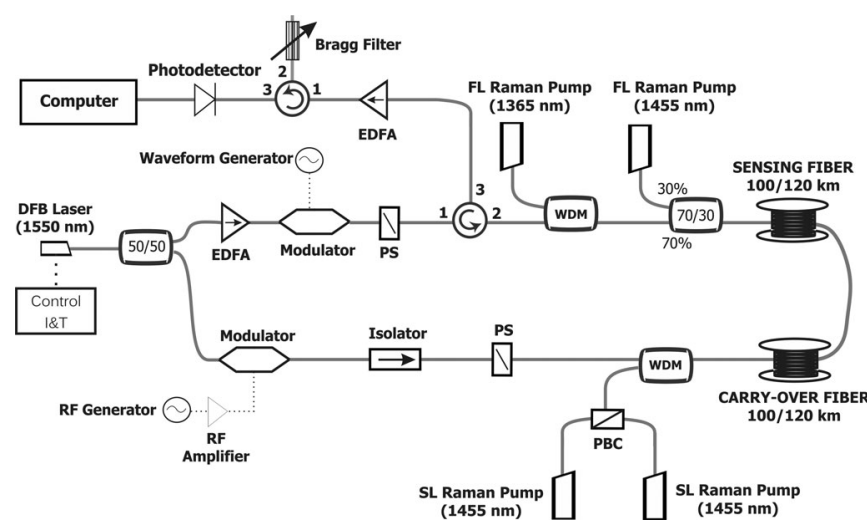

Fig. 2. Experimental setup of the implemented BOTDA sensor based on seeded second-order Raman assistance and pulse coding. DFB: distributed feedback laser; EDFA: erbium-doped fiber amplifier; PS: polarization switch; WDM: wavelength division multiplexer; RF: radio-frequency; PBC: polarization beam combiner: SL: semiconductor laser; FL: fiber laser.

the following pulses from the same sequence is expected. As a consequence, eventual Raman gain saturation does not distort the pulse sequences as occurs when the EDFA gain saturates.

As a difference to previously reported works [11], [19], here a suitable power optimization has to be carried out for very long sensing/carry-over fibers. As it will be later described in Section II-D, the presented procedure mainly pays attention to the Raman amplification of the probe waves to avoid effects such as amplified spontaneous Brillouin scattering, which might unbalance the power of both probe sidebands along the carry-over fiber. In addition, the limited extinction ratio of the pump pulses provides a $\mathrm{CW}$ component that amplifies/deplete the probe waves, increasing the possibilities to unbalance the power of both probe waves. This turns out to be critical for systems aiming at an extremely long remote sensing, since an eventual power imbalance of the probe sidebands would induce pump depletion along the sensing fiber and distort the measured Brillouin gain spectrum.

\section{Experimental Setup}

Fig. 2 shows the experimental setup implemented to realize extremely long-range distributed measurements. Two different experiments have been carried out using the same setup: $i$ ) the first experiment corresponds to BOTDA measurements along $100 \mathrm{~km}$ sensing distance, using a $200 \mathrm{~km}$ fiber-loop and a spatial resolution of $3 \mathrm{~m}$; and ii) the second one consists in the use of a $240 \mathrm{~km}$ fiber-loop for measurements along a $120 \mathrm{~km}$ range with $5 \mathrm{~m}$ spatial resolution.

A distributed feedback (DFB) laser operating at $1550 \mathrm{~nm}$ is the only optical source needed for the generation of Brillouin interacting signals in the system. Using a 50/50 coupler, the CW light from the laser is split into distinct branches to generate the coded pump signal and the probe signal.

A high-power pump signal is generated using an EDFA and an electro-optic modulator (EOM), which is used for on-off modulation of the $\mathrm{CW}$ laser light according to the Simplex code sequences. Note that the EDFA is placed before the EOM in order to avoid distortions in the pulse sequences resulting from the Erbium-doped fiber gain depletion [17]. To induce Raman amplification along the optical fiber, the coded pulse sequences are coupled with two depolarized fiber Raman lasers: a low-power seed first-order Raman pump at $1455 \mathrm{~nm}$ and a high-power second-order Raman pump at $1365 \mathrm{~nm}$. Although a $70 / 30$ coupler has been used here to couple the $1455 \mathrm{~nm}$ Raman pump, a WDM could have also been used instead to minimize coupling losses.

As far as the probe signal is concerned, this is obtained using the well-known double-sideband technique [23], in which two equally spaced sidebands are generated by intensity modulation of the CW laser light. Thus, by adjusting the RF modulation frequency on the EOM, the Brillouin gain spectrum can be easily scanned. To minimize the polarization-dependent gain (PDG) of the distributed Raman amplification along the fiber, two orthogonally-multiplexed low-RIN semiconductor lasers at $1455 \mathrm{~nm}$ have been coupled with the probe signal using a Raman pump unit that provides a PDG lower than $0.5 \mathrm{~dB}$.

The optical fiber consists of several drums of standard singlemode fiber, with a total length of $200 \mathrm{~km}$ or $240 \mathrm{~km}$ (depending on the experiment). Polarization diversity schemes, based on polarization switches (PS), have been used in both pump and probe branches to reduce the polarization dependence of the Brillouin gain spectrum (BGS) measurements.

Before the receiver, an EDFA operating in linear-gain regime is inserted to increase the power level of the coded probe signal reaching the photo-detector. A narrow-band fiber Bragg grating is used to select one of the probe sidebands and to filter out other unwanted frequency components, such as the amplified spontaneous emission noise from the EDFA, the Rayleigh signal generated by the coded Brillouin pump, the residual carrier of the probe and the second probe sideband. The data acquisition is carried through an acquisition card connected to a computer.

It is worth noticing that the implementation of optical pulse coding together with Raman amplification requires only small modifications of the standard BOTDA sensor; actually, most part of the setup in Fig. 2 is similar to the conventional BOTDA scheme. There are basically 2 differences with respect to a standard setup. The first one is the use of a programmable waveform generator to obtain the different coded pulse sequences in this case (instead of using a single-pulse generator). The second difference is related to the inclusion of three Raman pumps, added to the setup in Fig. 2 to provide distributed Raman amplification. Actually no optical repeaters with remotely powered elements have been added to the sensing fiber; thus, increasing the reliability of the system.

\section{Optimization of Power Levels in Long-Range BOTDA Sensors Using a Linear Sensing Fiber Configuration}

In order to avoid Brillouin pump depletion and nonlinear effects in the fiber, the power of the Raman pumps, Brillouin pump and probe signals have been carefully adjusted.

Although the use of seeded Raman amplification has been widely studied in the context of optical networks and wavelength division multiplexed (WDM) systems [14], [15], this is the first use of this kind of amplification in BOTDA sensors. In the 


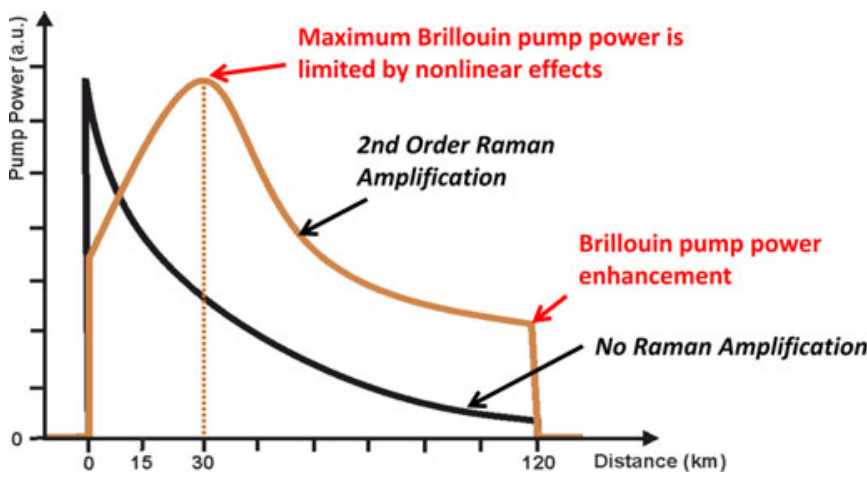

Fig. 3. Qualitative scheme showing the evolution of the Brillouin pump along the sensing fiber, as expected from the power optimization of the seeded secondorder Raman amplification.

context of distributed Brillouin sensing, the design and power optimization of a Raman amplifier has to deal with completely different conditions when compared with WDM transmissions systems. In particular, while in transmission systems the power of the signal is maintained at a very low level to avoid problems related to double Rayleigh scattering and cross-gain modulation resulting from Raman pump depletion [14], [15], in distributed Brillouin sensors, the Brillouin pump power has to be amplified up to the highest possible level in order to maximize its output power, but maintaining the highest pump power level in the fiber below the onset of nonlinear effects. Actually, the use of coded RZ-pulse sequences or single pump pulses with very low repetition rate makes typical levels of Raman pump depletion to have only a negligible impact on the performance of BOTDA sensors.

In this paper the optimization procedure for the forward propagating signals is carried out experimentally by knowing that, as a result of the second-order Raman amplification, the maximum Brillouin pump power occurs at about $30-40 \mathrm{~km}$ distance, as shown qualitatively in Fig. 3. This way, by monitoring the power of the Simplex-coded Brillouin pump at the far fiber end, the maximum Brillouin pump power along the fiber can be estimated (after characterizing the fiber attenuation), so that the power of the Brillouin and forward-propagating Raman pumps can be adjusted to maximize the Brillouin pump power at the end of the sensing fiber, but at the same time to avoid that the maximum power exceeds the threshold of nonlinear effects while propagating inside the fiber. Since the sensing fiber in this case corresponds to a standard single-mode fiber, the maximum Brillouin pump power allowed in the system is limited by the threshold of modulation instability, which is about 100$200 \mathrm{~mW}$ [20]. Thus, following the above-described procedure, the optimal condition has been achieved using a peak Brillouin pump input power of $7.5 \mathrm{dBm}$ and forward-propagating Raman pumps of $16.0 \mathrm{dBm}$ and $29.8 \mathrm{dBm}$ for the first- and second-order Raman pumps respectively. Note that power levels are referred at the input of the sensing fiber.

On the other hand, the backward-propagating Raman pump is adjusted to provide the highest possible probe power level at the end of the carry-over fiber (i.e. at the most distant sensing point, as shown qualitatively in the scheme of Fig. 4), but

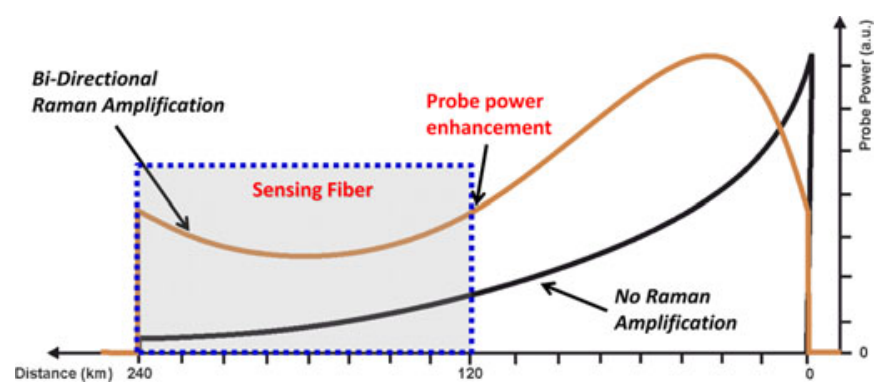

Fig. 4. Qualitative scheme showing the evolution of the probe signal along the entire optical fiber loop, as expected from the power optimization of the backward first-order Raman amplification.

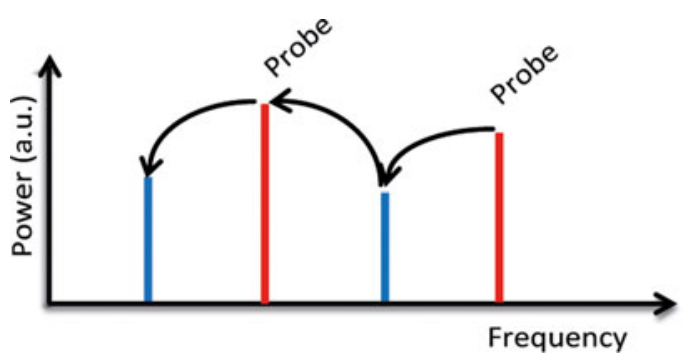

Fig. 5. Power imbalance resulting from the stimulated Brillouin scattering process occurring in a very long carry-over fiber when a high-power two-sideband probe signal is used. Power is transferred from higher to lower frequency components. Red lines represent the two probe waves propagating in backward direction (with respect to the Brillouin pump), and the blue lines correspond to the counter-propagating Brillouin components (in forward direction), initially generated by spontaneous Brillouin process.

at the same time to make pump depletion negligible along the sensing section [22]. Although this procedure seems to be similar to the one required in standard Raman-assisted BOTDA sensors [19], in this case the maximum probe power has to be reached within the carry-over fiber and not in the sensing fiber as in the usual fiber-loop case. Under such a condition, and in order to compensate the high attenuation of a long optical fiber, very high probe power levels are required within the carry-over fiber. Contrarily to the standard fiber configuration [19], the pump depletion that could occur in the carry-over fiber is not a problem for this long linear fiber arrangement, since potential variations of the probe power in this fiber section are not measured in the time-domain Brillouin traces. However, with high $\mathrm{CW}$ probe power levels another problem could arise in the long linear fiber configuration: this is related to the $\mathrm{CW}$ interaction of the two probe sidebands and their respective spontaneous Brillouin scattering components. In particular, when the CW probe power increases beyond the threshold of amplified spontaneous Brillouin scattering, the energy transferred from the probe waves to their respective Stokes components could strongly unbalance the power among the two sidebands, as illustrated in Fig. 5. The figure points out that under nonlinear Brillouin interaction, the energy transferred from the high-frequency probe sideband to its Stokes component is expected to be transferred to the lower-frequency probe sideband, which in turn transfers energy to the lowest Stokes component. In very long remote sensing, it is expected that this process would take place only along the carry-over fiber (within the nonlinear effective length), inducing depletion of the probe sideband at the higher frequency, 
while the lower-frequency sideband would not be significantly affected due to Brillouin gain/loss compensation. This cascaded energy transfer might quickly unbalance the power of the two probe waves, and thus compromise the robustness of the twosideband probe technique against pump depletion within the useful sensing fiber section [12]. Therefore, maintaining the same power for both probe waves is actually the main requirement that has to be satisfied when the power levels of the backward propagating Raman pump and probe wave are optimized. Thus, the proper optimization of these two backward-propagating signals requires $i$ ) to increase the probe power as much as possible within the sensing fiber, but keeping it below the level that induces pump depletion, and ii) to avoid power imbalance among the two probe waves within the carry-over fiber by maintaining the probe power below the critical power for the onset of amplified spontaneous Brillouin scattering. In this way the probe power at the receiver can be maximized, while pump depletion is avoided along the sensing fiber. This condition has been achieved in our implementation using an input probe signal of $-12.9 \mathrm{dBm}$ per sideband and a backward-propagation Raman pump of $28.1 \mathrm{dBm}$. Note that these values correspond to the optimum power launched at the far end of the carry-over fiber for a $100 \mathrm{~km}$ sensing range (200 km fiber loop). However, for a $240 \mathrm{~km}$ fiber-loop, slightly higher power levels might be required to optimize the probe SNR at the receiver. Unfortunately, the low-RIN Raman pump module used in this experiment limits the maximum power launched into the carry-over fiber, so that only the optimal condition for a $100 \mathrm{~km}$ distance can be fully satisfied. As a result, the same power levels have been used for both experiments (i.e. for 200 and $240 \mathrm{~km}$ fiber loops).

\section{E. Mathematical Model}

In order to provide a better understanding of the Brillouin and Raman processes occurring in the proposed system, numerical simulations have been carried out and compared with experimental data shown in Sections III and IV.

From a numerical point of view, the Raman-amplification characteristics of the system can be defined through steady-state equations [10], [19]. The variations of the three Raman pumps and Brillouin pump along the optical fiber can be obtained by solving the following coupled equations [10], in which Rayleigh and spontaneous Raman scattering have been neglected, as well as the Brillouin-Raman pump depletion resulting from the lowpower Brillouin probe:

$$
\frac{d P_{1}^{+}(z)}{d z}=-\alpha_{1} P_{1}^{+}(z)-g_{R 12} \frac{\nu_{1}}{\nu_{2}} P_{1}^{+}(z)\left[P_{2}^{-}(z)+P_{2}^{+}(z)\right]
$$

$$
\begin{aligned}
\frac{d P_{2}^{+}(z)}{d z}= & -\alpha_{2} P_{2}^{+}(z)-g_{R 2 B} \frac{\nu_{2}}{\nu_{B}} P_{2}^{+}(z) P_{B}^{+}(z) \\
& +g_{R 12} P_{1}^{+}(z) P_{2}^{+}(z) \\
\frac{d P_{2}^{-}(z)}{d z}= & \alpha_{2} P_{2}^{-}(z)+g_{R 2 B} \frac{\nu_{2}}{\nu_{B}} P_{2}^{-}(z) P_{B}^{+}(z) \\
& -g_{R 12} P_{1}^{+}(z) P_{2}^{-}(z)
\end{aligned}
$$

$$
\frac{d P_{B}^{+}(z)}{d z}=-\alpha_{B} P_{B}^{+}(z)+g_{R 2 B} P_{B}^{+}(z)\left[P_{2}^{-}(z)+P_{2}^{+}(z)\right]
$$

where the subscripts 1 and 2 represent Raman pumps at 1365 and $1455 \mathrm{~nm}$, respectively, the subscript $B$ denotes the wavelength of the Brillouin pump/probe (which can be considered identical from the point of view of Raman interaction), the superscripts + and - represent forward or backward propagation respectively, $\alpha_{i}$ is the fiber attenuation at the wavelength $i$, and $g_{R i j}$ is the Raman gain coefficient between the wavelengths $i$ and $j$.

On the other hand, the evolution of the Brillouin probe power $P_{B}^{-}(z)$ in the absence of the Brillouin pump in the Ramanamplified link can be obtained as:

$$
\frac{d P_{B}^{-}(z)}{d z}=\alpha_{B} P_{B}^{-}(z)+g_{R 2 B} P_{B}^{-}(z)\left[P_{2}^{-}(z)+P_{2}^{+}(z)\right] .
$$

Solving numerically the system of (1)-(4), the Brillouin pump power evolution along the fiber $P_{B}^{+}(z)$ can be obtained. With this information, the pump spectral broadening resulting from selfphase modulation (SPM) can be evaluated along the fiber [24]. Since the impact of SPM in BOTDA sensing depends on the pulse shape [24], a proper pulse shaping has to be employed for the simulations. In this case, a 4th-order super-Gaussian pulse has been used. Thus, in order to provide an estimation of the effective Brillouin gain bandwidth evolution along the fiber, the resulting pump spectrum has to be convolved with the natural Brillouin gain profile. This gain bandwidth can then be used to correct the Brillouin gain coefficient obtained for a non-broadened signal, resulting in a distance-dependent $\mathrm{g}_{B}(z)$.

Then, the Brillouin gain trace can be calculated as

$$
\text { Gain }(z)=\frac{g_{B}(z)}{A_{\text {eff }}} \frac{c T}{2 n} P_{B}^{+}(z)
$$

where $c$ is the speed of light, $T$ is the pulse duration, $n$ is the refractive index, and $A_{\text {eff }}$ is the fiber effective area.

\section{FIRST EXPERIMENT: BOTDA SENSING USING A $200 \mathrm{KM}$ Fiber LoOP AND A Spatial RESOlUtion of 3 M}

\section{A. Measurement Conditions}

The first experiment uses of a $200 \mathrm{~km}$-long optical fiber-loop, making distributed measurements possible up to a remote point located $100 \mathrm{~km}$ away from the interrogating unit. In particular, the $100 \mathrm{~km}$ sensing section (first fiber half) is composed of two $50 \mathrm{~km}$ fiber drums (named as Fiber A and B in the figures hereafter) with similar Brillouin frequency shifts (BFS $=10.66 \mathrm{GHz}$ at room temperature and zero strain). In order to clearly visualize the end of the sensing fiber at $100 \mathrm{~km}$ distance, a carry-over fiber (corresponding to the second $100 \mathrm{~km}$ fiber section) has been used showing a completely different BFS $(\sim 10.85 \mathrm{GHz})$. The uniformity of the BFS along the entire sensing fiber actually leads to a continuous SBS interaction between the coded Brillouin pump and the probe signal over $100 \mathrm{~km}$, resulting in the most critical situation to evaluate the impact of pump depletion on the system performance [12].

RZ Simplex coding with 127 bit has been implemented in this case, offering an SNR enhancement (so-called coding gain) of $7.5 \mathrm{~dB}$ with respect to the single pulse case [16]. The whole bit 


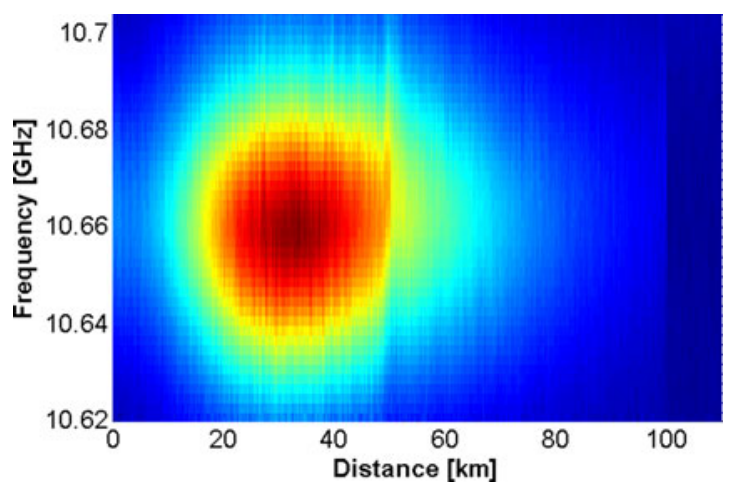

Fig. 6. Decoded BGS as a function of distance along $100 \mathrm{~km}$ sensing fiber, using a spatial resolution of $3 \mathrm{~m}$ and a $200 \mathrm{~km}$-long fiber loop.

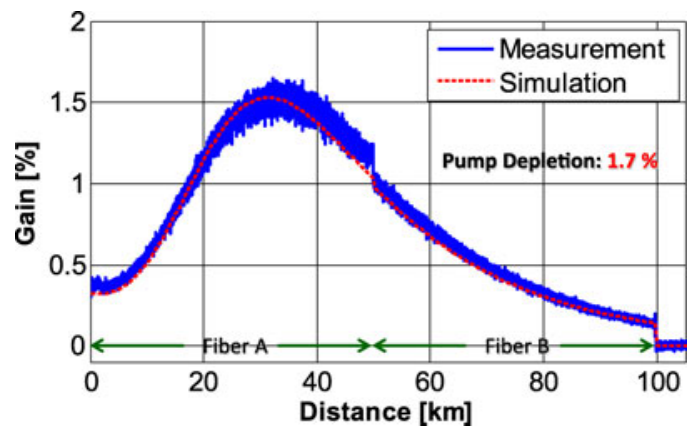

Fig. 7. Brillouin gain profile at $10.66 \mathrm{GHz}$ versus distance when a $200 \mathrm{~km}-$ long fiber loop is used with $3 \mathrm{~m}$ spatial resolution. The measured trace (blue straight line) is compared with the simulated trace (red dashed line).

duration is set to $100 \mathrm{~ns}$, while the single pulse duration is set to $30 \mathrm{~ns}$ (corresponding to a duty cycle of $30 \%$ ), resulting in a $3 \mathrm{~m}$ spatial resolution. Each coded BOTDA trace has been averaged 16 times, resulting in 2032 equivalent time-averaged traces for each scanned frequency.

\section{B. Distributed Measurements Along $100 \mathrm{~km}$}

After measuring the BOTDA coded traces, a linear decoding process is used to retrieve the single-pulse fiber response at the different scanned frequencies. Fig. 6 shows a top view of the decoded BGS as a function of the distance along a sensing range of $100 \mathrm{~km}$ when a spatial resolution of $3 \mathrm{~m}$ is used. By carefully inspecting the trace at the maximum Brillouin gain (i.e. at $10.66 \mathrm{GHz}$ ), shown in Fig. 7, the sensing fiber end at $100 \mathrm{~km}$ distance can clearly be seen, showing a Brillouin net gain of $\sim 0.17 \%$. In this figure, the experimental trace (blue continuous line) is compared with the BOTDA trace resulting from simulations at the peak Brillouin gain (red dashed line) using (1)-(6). A good agreement between theoretical and experimental results is observed.

By fitting the decoded gain spectra at every fiber position, the BFS along the entire sensing range is obtained, as shown in Fig. 8. The uncertainty in the measurement (related to the temperature and strain resolution) has been evaluated by calculating the standard deviation of the BFS over the last meters of fiber. In this case the calculated standard deviation is $1.5 \mathrm{MHz}$

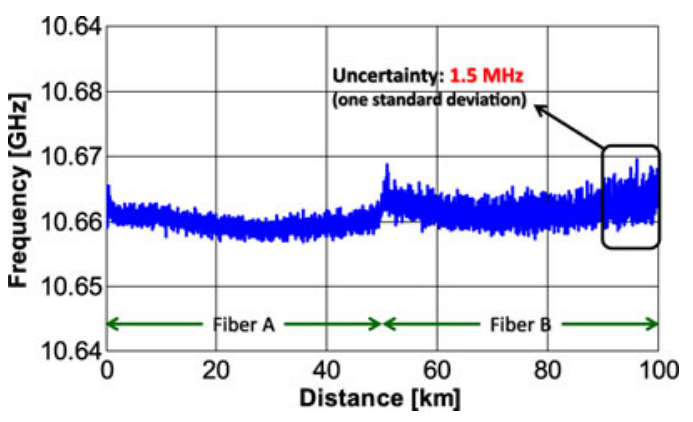

Fig. 8. BFS profile along $100 \mathrm{~km}$ distance, exhibiting a frequency uncertainty of $1.5 \mathrm{MHz}$ at the end of the sensing fiber.

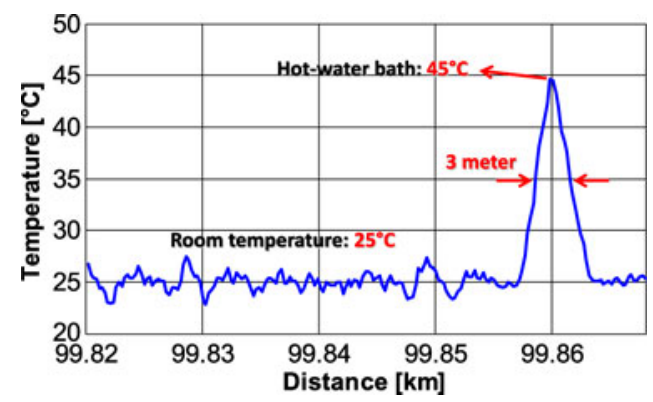

Fig. 9. Detection of a $3 \mathrm{~m}$ hot-spot at a $100 \mathrm{~km}$ sensing distance.

close to a $100 \mathrm{~km}$ distant position, which is equivalent to either a temperature resolution of $1.5 \mathrm{~K}$ or a strain resolution of $30 \mu \varepsilon$.

Pump depletion is finally evaluated measuring the residual coded Brillouin pump power with and without SBS interaction after propagation over $100 \mathrm{~km}$. Measurements indicate that the pump depletion is kept as low as $1.7 \%$, having a negligible impact on the BFS measurements [12]. Actually, using independent measurements, it has been verified that the small variations in the BFS observed in Fig. 8 are inherent to the sensing fiber spools, and do not correspond to distortions in the measurements due to potential pump depletion [12] or nonlinear effects [20], [24].

\section{Detection of a $3 \mathrm{~m} \mathrm{Hot-spot} \mathrm{at} 100 \mathrm{~km}$ Distance}

In order to verify the sensing capabilities of the implemented BOTDA sensor in such an extreme condition, 3 meters of fiber from the region showing the lowest SNR (i.e. near $100 \mathrm{~km}$ distance) are introduced into a hot-water bath at $45^{\circ} \mathrm{C}$, while the rest of the fiber is kept in a controlled room temperature at $25^{\circ} \mathrm{C}$.

Fig. 9 shows the temperature profile measured in the last meters of fiber, where it can be observed that the measured temperature profile perfectly matches the expected result. It is worth mentioning that this corresponds to the temperature measurement of a hot-spot located at a real $100 \mathrm{~km}$ distance away from the interrogation unit, in contrast with previously reported works [9], [19] where the far end of the fiber is typically located in the close vicinity of the sensor unit. 


\section{SECOND EXPERIMENT: BOTDA SENSING USING A 240 KM Fiber LoOP AND A SPATIAL RESOLUTION OF $5 \mathrm{M}$}

\section{A. Measurement Conditions}

In the second experiment a $240 \mathrm{~km}$-long fiber-loop has been implemented, making measurements possible up to a distance $120 \mathrm{~km}$ away from the interrogation unit. In addition to the fiber drums of the previous experiment, two $20 \mathrm{~km}$ fiber drums have been appended to the fiber link; one segment with a BFS equal to $10.68 \mathrm{GHz}$ (named as Fiber $\mathrm{C}$ ) has been used at the beginning of the sensing fiber, while another drum with a BFS close to $10.9 \mathrm{GHz}$ has been used to extend the carry-over fiber. Note that although sensing fibers with slightly different BFS have been used in this experiment, the frequency difference (up to $25 \mathrm{MHz}$ ) is within the Brillouin gain spectral width, and therefore a continuous Brillouin interaction occurs along the entire $120 \mathrm{~km}$-long sensing fiber. Under this condition, energy from the pump is efficiently transferred to the probe, maximizing pump depletion to provide the best validation of the proposed method.

Note that only $20 \mathrm{~km}$ have been added to the sensing range; however, this fiber extension reduces the SNR of the measurements by $12 \mathrm{~dB}$ with respect to the previous experiment. This comes from the fact that the pump power propagates along an extra $20 \mathrm{~km}$ of fiber length, i.e. being attenuated by an additional $4 \mathrm{~dB}$ at the end of the sensing fiber; while the probe signal propagates along an additional $40 \mathrm{~km}$ fiber length, i.e. being attenuated by another $8 \mathrm{~dB}$ before reaching the receiver. In order to recover part of this SNR reduction, the code length has been increased up to 255 bits and the spatial resolution has been downgraded to $5 \mathrm{~m}$. Note that, even though longer code length could in principle be used, some effects on polarization noise restricted the code length to 255 bits, as described below in the experimental results.

In order to enhance the SNR while maintaining a number of acquisitions similar to the previous experiment, each coded BOTDA trace in this case has been averaged 8 times, resulting in a total equivalent averaging number of 2040 traces per scanned frequency. Under this condition (i.e. with similar measurement time), the SNR is expected to be enhanced by $1.5 \mathrm{~dB}$ with respect to the previous experiment using $127 \mathrm{bit}$; this represents a net coding gain of $9 \mathrm{~dB}$ when compared to the conventional single pulse method [16].

\section{B. Distributed Measurements Along $120 \mathrm{~km}$}

After decoding the BOTDA traces, the BGS has been obtained as a function of distance along $120 \mathrm{~km}$. Fig. 10 shows a top view of the measured BGS when using a spatial resolution of $5 \mathrm{~m}$. It is important to point out that despite the extremely long optical fiber used in this case, it is still possible to distinguish the end of the sensing fiber at $120 \mathrm{~km}$, as clearly observed by inspecting Fig. 11. This figure also shows a comparison between the experimental BOTDA trace (blue continuous line) at $10.66 \mathrm{GHz}$ and the trace obtained by numerical simulations (red dashed line) using (1)-(6). A good matching between both curves is clearly observed again. The discontinuity at about $20 \mathrm{~km}$ dis-

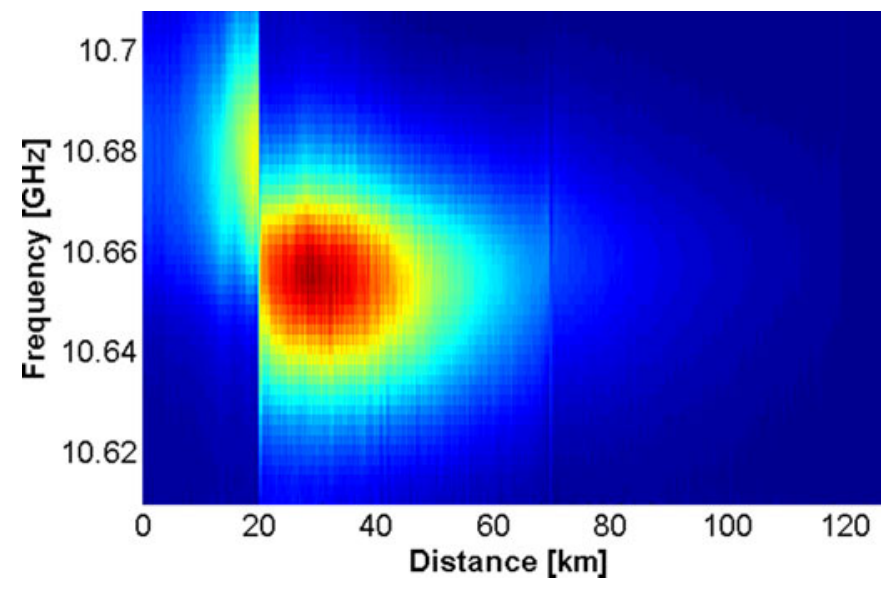

Fig. 10. Decoded BGS as a function of distance along $120 \mathrm{~km}$ sensing fiber, using a spatial resolution of $5 \mathrm{~m}$ and a $240 \mathrm{~km}$-long fiber loop.

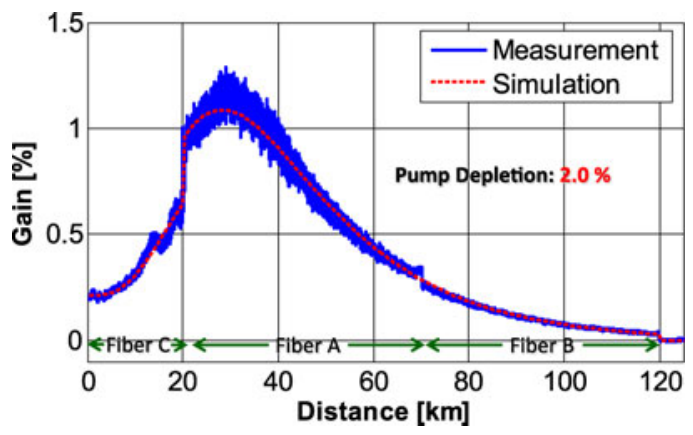

Fig. 11. Brillouin gain profile at $10.66 \mathrm{GHz}$ versus distance when a $240 \mathrm{~km}-$ long fiber loop is used with $5 \mathrm{~m}$ spatial resolution. Measurement (blue straight line) is compared with simulated trace (red dashed line).

tance is simply a result from the BFS difference between the fiber segments. The SNR at $120 \mathrm{~km}$ has been measured to be $\sim 6.5 \mathrm{~dB}$, corresponding to a Brillouin gain of $0.03 \%$. Thus, the obtained SNR at $120 \mathrm{~km}$ distance is high enough to provide reliable measurements of temperature- and strain-dependent variations of the BGS.

Although two polarization switches (one at pump and another at the probe sides) are used to minimize the impact of Brillouin PDG, measurements indicate that BOTDA traces turn out to be slightly affected by polarization noise when extremely long sensing fibers are used. Actually, it has been found that the used polarization diversity scheme, very common in standard BOTDA sensors, does not perform as expected with such a long optical fiber link (i.e. with $240-\mathrm{km}$ fiber loop). It is believed that this issue results from residual polarization noise coming from the finite extinction ratio of the two polarization switches. This may lead to non-orthogonal states of polarization after propagation over several tens of $\mathrm{km}$, inducing a residual Brillouin PDG in the measured BOTDA traces. In particular, it is observed that the residual polarization noise rises when the Brillouin gain increases (i.e. when longer code sequences are used), and has a larger impact at $30 \mathrm{~km}$ distance where the Brillouin pump reaches its maximum power, as shown in Fig. 11. This issue limits the maximum amplitude that the Brillouin gain can reach 


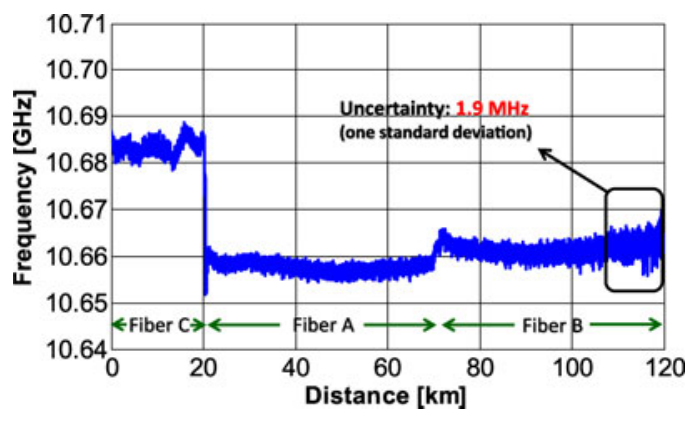

Fig. 12. BFS profile along $120 \mathrm{~km}$ distance, exhibiting a frequency uncertainty of $1.9 \mathrm{MHz}$ at the end of the sensing fiber.

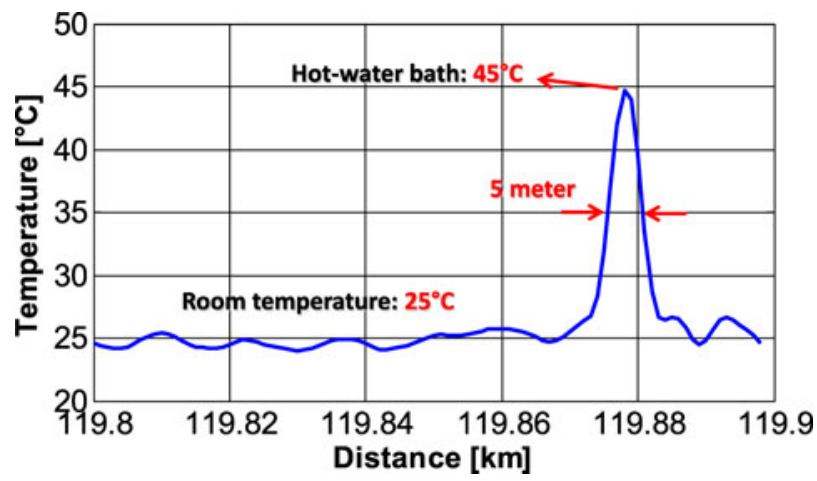

Fig. 13. Detection of a $5 \mathrm{~m}$ hot-spot at a $120 \mathrm{~km}$ sensing distance.

along the fiber, resulting in a real limitation to the maximum number of bits that can be used in the coded sequences.

The BFS along the fiber has been obtained by fitting the BGS at every fiber location, as shown in Fig. 12. It can be observed that using a pulse sequence of 255 bits, the polarization noise has a negligible impact on the BFS estimation. The standard deviation over the last meters of fiber (i.e. at the lowest SNR region) has been calculated to be $1.9 \mathrm{MHz}$, corresponding to a temperature/strain resolution of $1.9 \mathrm{~K} / 38 \mu \varepsilon$ at $120 \mathrm{~km}$ distance.

As in the previous experiment, the pump depletion is estimated by measuring the power difference of the residual coded Brillouin pump with and without Brillouin interaction. The pump depletion in this case is calculated to be $2.0 \%$, which is low enough to induce no biasing on the BFS measurements [18]. As in the previous case, using independent measurements here it has been also verified that the small variations in the BFS reported in Fig. 12 are inherent to the sensing fiber, and could be result of additional strain induced in the fiber during the coiling process or simply during fiber manufacturing.

\section{Detection of a $5 \mathrm{~m}$ Hot-spot at $120 \mathrm{~km}$ Distance}

Finally, measurements under extreme SNR conditions have been carried out; in this case a section of $5 \mathrm{~m}$ of fiber is heated up to $45^{\circ} \mathrm{C}$ at a $120 \mathrm{~km}$ distance, while the rest of the fiber is maintained at a controlled room temperature of $25^{\circ} \mathrm{C}$. Fig. 13 shows the measured temperature profile for the last $100 \mathrm{~m}$ of fiber, where it can be clearly observed the $5 \mathrm{~m}$ hot-spot at $45^{\circ} \mathrm{C}$. It is important to mention that this result constitutes the first demonstration of distributed measurements at a real $120 \mathrm{~km}$ distance away from the interrogating unit using a BOTDA sensor with no repeaters along the entire optical fiber.

\section{CONCLUSION}

In this paper a method to considerably extend the sensing range of BOTDA sensors has been presented. Using RZ optical pulse coding and seeded second-order Raman amplification, with a proper Raman pump power adjustment, extreme longrange BOTDA sensing capabilities have been reported along a real physical remote distance of 100/120 km from the sensor unit, using a 200/240 km-long entirely passive fiber-loop and $3 / 5 \mathrm{~m}$ spatial resolution. Such distances can be found while monitoring for instance long terrestrial pipeline or subsea flowlines for which no electrical power is available for repeater based sensing schemes. To the best of our knowledge this is the first time that such a distant remote sensing is reached using a BOTDA sensor, which can be considered as a significant breakthrough in the field of distributed optical fiber sensors.

The presented analysis as well as the obtained experimental results point out that the power budget of both Brillouin pump and probe waves must be carefully controlled when using a ultra-long linear fiber configuration to avoid exceeding the critical powers for modulation instability and amplified spontaneous Brillouin scattering. Thus, the optimization of the Raman pumps is critical to maximize the sensor performance, avoiding nonlinear effects in the fiber.

Finally, it is believed that further extension of the sensing range needs a novel conceptual step since the implemented system shows very little margin to increase power levels (including the Raman pump power) and to extend the code length.

\section{REFERENCES}

[1] T. Horiguchi, T. Kurashima, and M. Tateda, "A technique to measure distributed strain in optical fibers," IEEE Photon. Technol. Lett., vol. 2, no. 5, pp. 352-354, May 1990.

[2] T. Kurashima, T. Horiguchi, and M. Tateda, "Distributed-temperature sensing using stimulated Brillouin scattering in optical silica fibers," Opt. Lett., vol. 15, no. 10, pp. 1038-1040, 1990.

[3] T. Horiguchi, K. Shimizu, T. Kurashima, M. Tateda, and Y. Koyamada, "Development of a distributed sensing technique using Brillouin scattering," J. Lightw. Technol., vol. 13, no. 7, pp. 1296-1302, Jul. 1995.

[4] M. Niklès, L. Thévenaz, and P. Robert, "Simple distributed fiber sensor based on Brillouin gain spectrum analysis," Opt. Lett., vol. 21, no. 10, pp. 758-760, 1996.

[5] Q. Cui, S. Pamukcu, A. Lin, W. Xiao, D. Herr, J. Toulouse, and M. Pervizpour, "Distributed temperature sensing system based on rayleigh scattering BOTDA," IEEE Sensors J., vol. 11, no. 2, pp. 399-403, Feb. 2011.

[6] X. Zhang, J. Hu, and Y. Zhang, "A hybrid single-end-access BOTDA and COTDR sensing system using heterodyne detection," J. Lightw. Technol., vol. 31, no. 12, pp. 1954-1959, Jun. 2013.

[7] Y. Dong, L. Chen, and X. Bao, "Extending the sensing range of brillouin optical time-domain analysis combining frequency-division multiplexing and in-line EDFAs," J. Lightw. Technol., vol. 30, no. 8, pp. 1161-1167, Apr. 2012.

[8] M. A. Soto, G. Bolognini, and F. Di Pasquale, "Long-range simplex-coded BOTDA sensor over $120 \mathrm{~km}$ distance employing optical preamplification," Opt. Lett., vol. 36, no. 2, pp. 232-234, 2011.

[9] X. Angulo-Vinuesa, S. Martin-Lopez, J. Nuno, P. Corredera, J. D. AniaCastanon, L. Thevenaz, and M. Gonzalez-Herraez, "Raman assisted brillouin distributed temperature sensor over $100 \mathrm{~km}$ featuring $2 \mathrm{~m}$ resolution and $1.2^{\circ}$ C uncertainty," J. Lightw. Technol., vol. 30, no. 8, pp. 1060-1065, Apr. 2012. 
[10] S. Martin-Lopez et al., "Brillouin optical time-domain analysis assisted by second-order Raman amplification," Opt. Exp., vol. 18, no. 18, pp. 1876918778, 2010.

[11] M. A. Soto, G. Bolognini, and F. Di Pasquale, "Optimization of longrange BOTDA sensors with high resolution using first-order bi-directional Raman amplification," Opt. Exp., vol. 19, no. 5, pp. 4444-4457, 2011.

[12] L. Thévenaz, S. F. Mafang, and J. Lin, "Effect of pulse depletion in a Brillouin optical time-domain analysis system," Opt. Exp., vol. 21, no. 12, pp. 14017-14035, 2013.

[13] D. M. Nguyen et al., "Sensitivity enhancement in long-range distributed Brillouin fiber sensor using an anti-Stokes single-sideband probe and a bidirectional EDFA," in Proc. Photon. Global Conf., 13-16, Dec. 2012, pp. 1-4.

[14] S. Faralli, G. Bolognini, G. Sacchi, S. Sugliani, and F. Di Pasquale, "Bidirectional higher order cascaded Raman amplification benefits for $10-\mathrm{Gb} / \mathrm{s}$ WDM unrepeated transmission systems," J. Lightw. Technol., vol. 23, no. 8, pp. 2427-2433, Aug. 2005.

[15] S. Faralli, G. Bolognini, M. A. Andrade, and F. Di Pasquale, "Unrepeated WDM transmission systems based on advanced first-order and higher order Raman-copumping technologies," J. Lightw. Technol., vol. 25, no. 11, pp. 3519-3527, Nov. 2007.

[16] M. D. Jones, "Using simplex codes to improve OTDR sensitivity," IEEE Photon. Technol. Lett., vol. 5, no. 7, pp. 822-824, Jul. 1993.

[17] M. A. Soto, G. Bolognini, F. Di Pasquale, and L. Thévenaz, "Long-range brillouin optical time-domain analysis sensor employing pulse coding techniques," Meas. Sci. Technol., vol. 21, no. 9, p. 094024, 2010.

[18] X.-H. Jia, Y.-J. Rao, K. Deng, Z.-X. Yang, L. Chang, C. Zhang, and Z.-L. Ran, "Experimental demonstration on 2.5-m spatial resolution and $1{ }^{\circ} \mathrm{C}$ temperature uncertainty over long-distance BOTDA with combined Raman amplification and optical pulse coding," IEEE Photon. Technol. Lett., vol. 23, no. 7, pp. 435-437, Apr. 2011.

[19] M. A. Soto, G. Bolognini, and F. Di Pasquale, "Simplex-coded BOTDA sensor over $120 \mathrm{~km}$ SMF with $1 \mathrm{~m}$ spatial resolution assisted by optimized bidirectional Raman amplification," IEEE Photon. Technol. Lett., vol. 24, no. 20, pp. 1823-1826, Oct. 2012.

[20] S. F. Mafang and L. Thévenaz, "Impact of Raman scattering and modulation instability on the performances of brillouin sensors," presented at the 21st Int. Conf. Opt. Fiber Sensors, Ottawa, Canada, 2011.

[21] C. R. S. Fludger, V. Handerek, and R. J. Mears, "Pump to signal RIN transfer in Raman fiber amplifiers," J. Lightw. Technol., vol. 19, no. 8, pp. 1140-1148, Aug. 2001.

[22] M. A. Soto, G. Bolognini, and F. Di Pasquale, "Analysis of pulse modulation format in coded BOTDA sensors," Opt. Exp., vol. 18, no. 14, pp. 14878-14892, 2010.

[23] M. Niklès, L. Thévenaz, and P. A. Robert, "Simple distributed fiber sensor based on brillouin gain spectrum analysis," Opt. Exp., vol. 21, no. 10, pp. 758-760, 1996.

[24] S. M. Foaleng, F. Rodríguez, S. Martin Lopez, M. González Herráez, and L. Thévenaz, "Detrimental effect of self-phase modulation on the performance of brillouin distributed fiber sensors," Opt. Lett., vol. 36, no. 2, pp. 97-99, 2011.
Xabier Angulo-Vinuesa was born in Bilbao, Spain, in 1982. He received the Electronics Engineer's degree from the University of the Basque Country, Basque Country, Spain, in September 2009. Since 2010, he has been working toward the Ph.D. degree in the Optics Institute at the Spanish Council for Research.

During 2008-2009 he was with the Interdisciplinary Nanoscience Centre at Aarhus University, Denmark, working on photonic crystal waveguides. His current research interests include nonlinear fiber optics and fiber optic sensors.

Sonia Martin-Lopez received the Ph.D. degree from the Universidad Complutense de Madrid, Madrid, Spain, in May 2006.

The topic of her doctoral dissertation was on experimental and theoretical understanding of continuous-wave pumped supercontinuum generation in optical fibers. She had a predoctoral stay in the Nanophotonics and Metrology Laboratory, Ecole Polytechnique Federale de Lausanne, Switzerland. She was a Postdoctoral researcher in the Applied Physics Institute and in the Optics Institute at the Spanish Council for Research for six years. She is currently a Postdoctoral Researcher in the Photonics Engineering Group, University of Alcala, Madrid, Spain. She is author or coauthor of more than 100 papers in international refereed journals and conference contribution. Her current research interests include nonlinear fiber optics and distributed optical fiber sensors.

Sang-Hoon Chin received the M.Sc. degree in information and communication from the Gwangju Institute of Science and Technology (GIST), Gwangju, Korea, in 2005, and the Ph.D. degree in electrical engineering from the Ecole Polytechnique Fédérale de Lausanne, Lausanne, Switzerland, in 2009, where he developed his expertise on slow and fast light, optical signal processing, distributed fiber sensing in optical fibers.

In 2010, he visited the Polytechnique University in Valencia, Spain, and Thales Research \& Technology, Palaiseau, France, and gained an expertise in microwave photonics. He joined Omnisens SA, Morges, Switzerland, in 2011.

Juan Diego Ania-Castañon obtained the M.Sc. degree in physics from the Universidad Complutense de Madrid, Madrid, Spain, and the Ph.D. degree in theoretical physics from the Universidad de Oviedo and Instituto de Estructura de la Materia, CSIC, Madrid, Spain.

In 2001, he joined the Photonics Research Group, Aston University, Birmingham, U. K., first as a Contract Research Fellow and then as an EPSRC Advanced Research Fellow from 2004. In 2007, he took an appointment as a Tenured Scientist at Instituto de Óptica, CSIC, Madrid, Spain. He has authored or coauthored more than 120 papers in refereed journals and international conferences. His main research interests deal with the exploitation of nonlinear effects in optical fiber.

Pedro Corredera received the B.Sc. and Ph.D. degrees in physics from the University of Salamanca, Salamanca, Spain, in 1985 and 1989, respectively.

In 1989, he joined the Institute of Optics, CSIC, where he worked on IR radiometry and optical fibres metrology. In 1990-91 he joined at National Physical Laboratory, Teddington, /U.K., working in cryogenic radiometry and optical fibre metrology. From 1996-2010, he was with the Institute of Applied Physics and he created a research group in Optical Communications Technologies (GiTCO) with three lines of work: optical fibers metrology, nonlinear properties of optical fibers and applications, and optical fiber sensors. In 2010, he rejoined the Institute of Optics in the group "nonlinear dynamics and fiber optics." He has published more than 60 articles in scientific and technical journals and 100 contributions at international conferences. His current research interests include fiber-optic measurements, optical fiber sensors, nonlinear fiber optics, and IR radiometry and detection.

Dr. Corredera is member of the Spanish Society of Optics, the Optical Society of America, and the European Optical Society.
Dr. Soto is member of the Optical Society of America, and he is in the Board of Reviewers of major international journals in fields of optical communications and photonics. 
Etienne Rochat (M'11) received the Bachelor's degree of engineer in microtechnology from the Swiss Technical College, Yverdon, Switzerland, in 1991, an engineering degree in microtechnology from the Swiss Federal Institute of Technology, Lausanne, Switzerland, in 1995, and the Ph.D. degree in science from the University of Neuchâtel, Neuchatel, Switzerland, in 2000.

From 1995 to 2000, he was a Graduate Research Assistant at the Institute of Microtechnology, University of Neuchâtel, within the group of Prof. Dändliker, where he mainly worked on rare-earth doped fibre sources for sensor applications. He also studied and developed high power doped fibre amplifiers for coherent space communication systems, within a project of the European Space Agency. From 2000 to 2001, he was a Research Scientist with ABB Corporate Research in Dättwil, Switzerland, developing fibre sensors for remote pressure and temperature measurement in oil reservoir; and from 2001 to 2004, he was a Research Fellow at the University of Essex, Colchester, U.K., working mainly on optical communication, including high power Raman amplifiers and in polarisation multiplexing capabilities of multimode fibres and AWGs for bandwidth improvement of access and metro networks. In 2004, he joined Omnisens SA, Morges, Switzerland, where he worked as Product Manager for the company Trace Gas Analysers, a now discarded product based on optical spectroscopy. In 2009 , he became R\&D manager for fibre optic distributed sensing and since 2010 is the Head of engineering, leading both new development on Omnisens Brillouin sensing technology and product development. He has authored more than 40 papers and three patents.

Miguel Gonzalez-Herraez received the M.Eng. and D.Eng. degrees from the Polytechnic University of Madrid, Madrid, Spain, in 2000 and 2004 respectively.

While working toward the D.Eng. degree, he worked first as a Research Assistant and then a Postdoctoral Fellow in the Applied Physics Institute at the Spanish Council for Research, Madrid, Spain, and had several long stays in the Nanophotonics and Metrology Laboratory, Ecole Polytechnique Federale de Lausanne, Switzerland. In October 2004, he was appointed an Assistant Professor in the Department of Electronics, University of Alcalá, Madrid, Spain, where he was promoted to an Associate Professor in June 2006. He is the author or coauthor of more than 120 papers in international refereed journals and conference contributions and has given several invited talks at international conferences. His research interests include the wide field of nonlinear interactions in optical fibers.

Dr. González-Herráez received several important recognitions to his research career, including the European Research Council Starting Grant and the "Agustin de Betancourt" prize of the Spanish Royal Academy of Engineering.
Luc Thévenaz (M'01-SM'12) received the M.Sc. and Ph.D. degrees in physics from the University of Geneva, Geneva, Switzerland.

In 1988, he joined the Swiss Federal Institute of Technology of Lausanne, Lausanne, Switzerland, where he currently leads a research group involved in photonics, namely fibre optics and optical sensing. Research topics include Brillouin-scattering fibre sensors, slow and fast light, nonlinear fibre optics and laser spectroscopy in gases. He achieved with his collaborators the first experimental demonstration of optically-controlled slow and fast light in optical fibres, realized at ambient temperature and operating at any wavelength since based on stimulated Brillouin scattering. He also contributed to the development of Brillouin distributed fibre sensing by proposing innovative concepts pushing beyond barriers. During his career he was with Stanford University, the Korea Advanced Institute of Science and Technology, Tel Aviv University, and the University of Sydney. In 2000, he cofounded the company Omnisens that is developing and commercializing advanced photonic instrumentation. He is an editor in two major scientific journals

Dr. Thévenaz is fellow of the Optical Society of America. 